\title{
BMY 14802, a Sigma Receptor Ligand for the Treatment of Schizophrenia
}

George R. Gewirtz, M.D., Jack M. Gorman, M.D., Jan Volavka, M.D., Joseph Macaluso, M.D., Greta Gribkoff, M.S., Duncan P. Taylor, Ph.D., and Richard Borison, M.D.

The theoretical role of sigma receptors in psychosis has led to the development of selective sigma receptor ligands as potential antipsychotic agents. BMY 14802 has its most potent binding at the sigma binding site, with some degree of serotonin subtype $1 A$ and negligible dopamine receptor binding. It is atypical of standard neuroleptics in that it does not induce catalepsy in rats. It has been shown to have efficacy in animal models of psychosis. It was hypothesized that the drug would have antipsychotic effects in humans without producing the extrapyramidal side effects typical of standard neuroleptics. We report here the results of an uncontrolled, multicenter safety and efficacy study of patients with acute exacerbations of schizophrenia treated with BMY 14802. After 1 week of single-blind placebo treatment, 28 patients were treated with BMY 14802 (up to $3000 \mathrm{mg} /$ day) for up to 4 weeks. There was no significant improvement in psychiatric symptoms, as measured by the total Brief Psychiatric Rating Scale scores or Clinical Global Improvement. There were no changes in involuntary movements, as measured by the Abnormal Involuntary Movement Scale, or in extrapyramidal symptoms as measured by the Simpson-Angus Scale. [Neuropsychopharmacology 10:37-40, 1994]
KEY WORDS: Sigma; Antipsychotic; Schizophrenia; BMY 14802

The demonstration that benzomorphans, such as SKF 10,047, produce "canine delirium" (Martin 1976) and psychosis in humans (Keats and Telford 1964) led to the proposal that distinct sigma receptors exist that mediate these effects. Binding studies using $\left[{ }^{3} \mathrm{H}\right] \mathrm{SKF}$ 10,047 (Su 1982; Largent 1986) and $(+)\left[{ }^{3} \mathrm{H}\right]-3-P P P$ (Gundlach 1986) subsequently identified and characterized sigma sites in guinea pig and rat brains. The distribution of sigma binding sites was determined in the post-mortem human brain (Weissman 1988) using

From the New York State Psychiatric Institute (GRG, JMG), Columbia University, Nathan Kline Institute (JV), New York University Medical College, Pilgrim Psychiatric Center (JM), Mount Sinai Medical College, New York, New York; Bristol-Myers Squibb Company (GG, DPT), Wallingford, Connecticut; and VA Medical Center (RB), Medical College of Georgia, Augusta, Georgia.

Address correspondence to: George R. Gewirtz, M.D., New York State Psychiatric Institute, 722 West 168th Street, New York, New York 10032.

Received March 15, 1993; accepted August 31, 1993. $\left[{ }^{3} \mathrm{H}\right.$ ]haloperidol in the presence of spiperone to block $\mathrm{D}_{2}$ dopamine receptor binding and found to be highest in the cerebellum, nucleus accumbens, and various regions of the cerebral cortex. These sites do not bind other opioids or dopamine and have a high affınity for haloperidol and other neuroleptics. Although early studies suggested that phencyclidine and sigma compounds might have a common binding site, they have since been demonstrated to be different.

Using the 2-deoxy- $D-\left[1-{ }^{14} \mathrm{C}\right]$ glucose method, regional central nervous system changes in glucose utilization with sigma ligands BMY 14802 and BW 234U (rimcazole) were demonstrated in the rat (della Puppa and London 1989). In addition, a recent post-mortem study found reduced densities of sigma receptors in brains of patients with schizophrenia (Weissman et al. 1991). The function of the sigma receptor is not known; however, one study suggested that it is coupled to phosphoinositol turnover in peripheral tissues.

The theoretical role of sigma receptors in psychosis has led to the development of selective sigma receptor ligands as potential antipsychotic agents. BMY 14802 
is one such agent. It is structurally related to the butyrophenones and buspirone. Its most potent receptor affinity is for the sigma receptor, with some degree of serotonin subtype $1 \mathrm{~A}$ but negligibledopamine receptor binding. The compound is also atypical of standard neuroleptics in that it does not induce catalepsy in rats. It has been shown to have efficacy in animal models of psychosis. It inhibits apomorphine-induced stereotypy in rats, apomorphine-induced climbing in mice, and the conditioned avoidance response in rats. In monkeys given chronic amphetamines, BMY 14802 partially antagonized increased checking and submissive gestures (Taylor et al. 1991). Chronic administration led to depolarization block of mesolimbic (A10) but not nigrostriatal (A9) dopamine cells, and, in contrast to haloperidol, long-term administration did not produce an increased number of $D_{2}$ receptor binding sites. It was therefore hypothesized that BMY 14802 would have antipsychotic effects in humans without producing the extrapyramidal side effects typical of standard neuroleptics.

\section{METHODS}

A multicenter, uncontrolled inpatient safety and efficacy study was conducted in subjects who met DSM III-R criteria for schizophrenia with an acute exacerbation. The protocol required the patients had not received depot neuroleptics for 2 months and oral antipsychotics for 7 days prior to receiving BMY 14802. Subjects had to meet the following severity of illness criteria: a Brief Psychiatric Rating Scale (BPRS) score of greater than or equal to 35 , with at least one of the following BPRS items: hallucinatory behavior, conceptual disorganization, unusual thought content, and suspiciousness (rated $\geqslant 4$ and the sum of the items $\geqslant 12$ ).

Other inclusion criteria were that subjects be male or infertile females, aged 21 to 55 years, and in good medical health. Exclusion criteria included significant head trauma with loss of consciousness, and significant recent psychoactive substance abuse or dependence.

For the first week, all subjects received single-blind placebo. Subjects whose BPRS total score did not meet the inclusion criteria at end of baseline or whose BPRS total score improved by $20 \%$ during the placebo phase were excluded from the study. For the following 28 days, subjects received active drug. Initially, the dosing schedule was $200 \mathrm{mg}$ to $500 \mathrm{mg}$ of active drug four times daily, up to a maximum of $2000 \mathrm{mg} /$ day. This dosing schedule was then revised, with subjects receiving $500 \mathrm{mg}$ to $1500 \mathrm{mg}$ twice daily up to a maximum of 3000 $\mathrm{mg} /$ day. Safety monitoring included daily vital signs and weekly electrocardiograms, clinical laboratory work (complete blood count, serum chemistries, and urinalysis). Weekly outcome measures included the BPRS,
Clinical Global Improvement(CGI), Abnormal Involuntary Movement Scale (AIMS), and Simpson-Angus Scale.

\section{RESULTS}

Twenty-eight subjects were enrolled, 27 men and one woman; their ages ranged from 22 to 48 years, with a mean age of 35.7. Fifteen subjects were white and 13 subjects were black. Twenty-seven patients met the entrance criteria for withdrawal of antipsychotic medication prior to receiving BMY 14802. One patient was allowed to continue thioridazine until the day before starting BMY 14802 because the investigator was concerned that the patient would severely decompensate if not receiving any medication. No patients received depot neuroleptics within 1 year of beginning the study. Thirteen subjects completed the study, 12 men and one woman. Of the completers, eight were white and five were black, their age range was 26 to 48 years, and the mean age was 37.2. Of the 15 subjects withdrawn from the study, the primary reason was lack of efficacy in six patients, adverse experience in five, withdrawal of patient consent in three, and cocaine use during the study in one.

The most frequent side effect was mild to moderate sinus tachycardia, seen in 12 patients. One subject was withdrawn from the study on day 6 due to frequent asymptomatic sinus tachycardia, although this subject also had tachycardia noted during the placebo period. Another subject was dropped from the study at week 3 due to asymptomatic tachycardia.

One subject was withdrawn at week 3 due to creatine phosphokinase (CPK) elevations. Mild to moderate CPK elevations were noted in seven patients, but this was not a standard test for safety monitoring, and the incidence is unknown.

Of the patients with CPK elevations who had isoenzymes performed, each was of skeletal muscle origin. In one patient, the CPK elevation was associated with a positive antinuclear antibodies and rheumatoid factor and a muscle biopsy showing individual myofiber necrosis. At baseline, this patient had myalgias and a low white count, but no CPK was obtained. One patient had a mild transient hyperkalemia. There were no other significant laboratory abnormalities. Other reported adverse experiences were headache, nausea, hypotension, orthostatic hypotension, hypertension, dizziness, syncope, bradycardia, agitation, sedation, and insomnia, all generally of mild or moderate intensity.

The pretreatment total BPRS score for all 28 patients was $47.82 \pm 6.41$ and the baseline day mean (at the end of placebo period) was $51.54 \pm 6.41$. There was a statistically significant mean increase. 


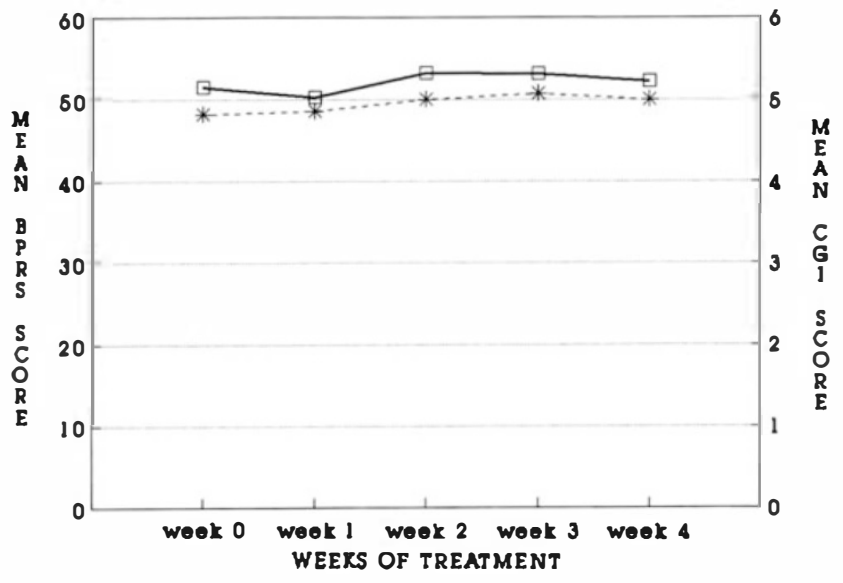

Figure 1. Mean BPRS and CGI scores for patients treated with BMY 14802, study weeks 0 to $4(n=28)$. Solid, boxed line $=$ BPRS. Dashed, starred line $=$ CGI.

The criteria for defıning a patient as improved was a reduction in the total BPRS score of greater than $20 \%$ from baseline. Six of the 28 patients met this criterion for improvement. On the CGI scale, one patient was rated as much improved (score $=2$ ), five patients were rated as minimally improved (score $=3$ ), with the rest rated as no change or worse.

An "intent-to-treat" analysis (Sackett and Gent 1979) was performed with all patients who were assessed at baseline and at least once more; those subjects who had missing scores had their last ratings carried forward. Mean total BPRS and CGI scores at each week of treatment are given in Figure 1. A repeatedmeasures analysis of variance (ANOVA) of BPRS scores showed no significant difference from baseline to weeks 1, 2, 3, and 4. Also using repeated-measures ANOVA, no significant differences were seen in CGI scores for these patients over time.

Repeated-measures ANOVA showed no significant difference from baseline in AIMS scores over the 4 weeks of treatment. Simpson-Angus Scale scores did show a significant change over time in Simpson-Angus scores from baseline to week $4(F=2.56 ; p<.05)$.

\section{DISCUSSION}

BMY 14802 was of particular interest as a potential antipsychotic because it represented a novel treatment approach with little propensity to cause extrapyramidal side effects. Although some patients showed mild to moderate global improvement, this study did not providecompelling evidence that BMY 14,802 would offer clinically significant efficacy.

There was some improvement in extrapyramidal symptoms during treatment. This could be attributable to an effect of BMY 14802, as catalepsy in rats was reversed by the drug, or to gradual decrease in dopamine blockade by residual prior antipsychotic agents.

The lack of clinically important therapeutic effects in this study raises the issue of whether sigma receptor ligands represent potential treatments for psychosis or schizophrenia. Rimcazole, another selective sigma receptor ligand, failed to show significant efficacy and was associated with possible increased risk of seizures (Chouinard and Annable 1984).

Much is still unknown about the sigma receptor. Classifying agents as agonists versus antagonists at the sigma site is not possible because a biologic function of the sigma binding site remains to be elucidated. Agents may be developed with properties that are significantly different from BMY 14802 that prove to be of clinical interest.

Other explanations for lack of efficacy in this study include: (1) the dose used was either too low or too high, animal studies suggest antipsychotic efficacy of BMY 14802 at lower doses per kilogram body weight than that used in this study; and (2) this drug may have a longer onset of action than could be demonstrated in a 4-week study.

Given that some patients showed improvement, there may be a subgroup of patients with schizophrenia who are responsive to sigma receptor agents, but the sample size of this study did not allow this to be definitively explored. Given the significant sigma binding of many effective dopamine blocking antipsychotic agents, BMY 14802 might have a role as an adjunct to other drugs, but this has not been attempted.

Interestingly, BMY 14802 was recently shown to prevent 1-methyl-4-phenyl-1,2,3,6-tetrahydropyridine and amphetamine-induced toxicity to dopamine neurons. It may be that such sigma receptor agents will not prove to be efficacious for acute psychotic episodes but may prevent damage to dopamineneurons that we have hypothesized may occur over time in schizophrenia.

The animal models used to assess the potential antipsychotic efficacy of BMY 14802 are now standardly used in the preclinical assessment of new antipsychotic drugs. However, the limitations of these models were demonstrated by their failure to predict the negative results of BMY 14802 in humans; reassessment of their use may well be warranted.

\section{REFERENCES}

Chouinard G, Annable L (1984): An early phase II clinical trial of BW $234 \mathrm{U}$ in the treatment of acute schizophrenia in newly admitted patients. Psychopharmacology 84:282284

della Puppa A, London ED (1989): Cerebral metabolic effects of sigma ligands in the rat. Brain Res 505:283-290

Gundlach AL, Largent BL, Snyder SH (1986): Autoradiographic localization of sigma receptor binding sites in 
guinea pig and rat central nervous system with $(+)^{3} \mathrm{H}-$ 3-(3-hydroxyphenyl)-N-(1-propyl)piperidine. J Neurosci 6:1757-1770

Keats AS, Telford J (1964): Narcotic antagonists as analgesics. Clinical aspects. In Gould RF, (ed), Molecular Modification in Drug Design, Advances in Chemistry Series 45. Washington, DC, American Chemical Society, pp 170176.

Largent BL, Gundlach AL, Snyder SH (1986): Pharmacological and autoradiographic discrimination of sigma and phencyclidine receptor binding sites in brain with $(+)$ $\left[{ }^{3} \mathrm{H}\right] \mathrm{SKF}$ 10.047, (+)-[ $\left.{ }^{3} \mathrm{H}\right]-3-[3-h y d r o x y p h e n y l]-\mathrm{N}-(1-$ propyl)piperidine and [ $\left.{ }^{3} \mathrm{H}\right]-1-[1-(2-$ thienyl)cyclohexyl]piperidine. J Pharmacol Exp Ther 739-748

Martin WR, Eades CG, Thompson JA, Huppler RE, Gilbert PE (1976): The effects of morphine and nalorphine-like drugs in the non-dependent and morphine-dependent chronic spinal dog. J Pharmacol Exp Ther 197:517-532

Sackett DL, Gent M (1979): Controversy in counting and at- tributing events in clinical trials. N Engl J Med 301: $1410-1412$

Su T (1982): Evidence for sigma opioid receptor: Binding of $\left[{ }^{3} \mathrm{H}\right] \mathrm{SKF}-10,047$ to etorphine-inaccessible sites in guineapig brain. J Pharmacol Exp Ther 223:284-290

Taylor DP, Eison MS, Moon SL, Yocca FD (1991): BMY 14802: A potential antipsychotic with selective affinity for sigmabinding sites. In Tamminga, CA and Schulz, SC (eds), Advances in Neuropsychiatry and Psychopharmacology, Vol 1: Schizophrenia Research. New York, Raven Press, pp 307-315

Weissman AD, Su T, Hedreen JC, London ED (1988): Sigma receptors in post-mortem human brains. JPharmacol Exp Ther 247:29-33

Weissman AD, Cassanova MF, Kleinman JB, London ED, De Souza EB (1991): Selective loss of cerebral cortical sigma, but not PCP binding sites in schizophrenia. Biol Psychiatry $29: 41-54$. 